\title{
Inflammatory Bowel Disease and COVID-19 Vaccination: A Patients' Survey
}

\author{
Bénédicte Caron $^{1} \cdot$ Elise Neuville ${ }^{1} \cdot$ Laurent Peyrin-Biroulet $^{1}{ }^{[0}$
}

Received: 26 March 2021 / Accepted: 5 May 2021 / Published online: 12 May 2021

(c) The Author(s), under exclusive licence to Springer Science+Business Media, LLC, part of Springer Nature 2021

\begin{abstract}
Background Vaccination against COVID-19 is a major public health challenge, including the community of patients with inflammatory bowel disease. Vaccination coverage is suboptimal in inflammatory bowel disease population. It is of paramount importance to ensure an effective and rapid vaccination program with the adherence of the largest number of well-informed patients.

Aims We assessed the acceptance of COVID-19 vaccination among inflammatory bowel disease patients.

Methods We performed a survey as part of routine practice, between January 8th and February 22nd, 2021. All consecutive adult patients followed at Nancy University Hospital for inflammatory bowel disease were included. Patients completed a self-administered, structured, paper-based questionnaire. Demographic data, medical history, knowledge, and perceptions of COVID-19 vaccination were collected.

Results Among the 104 patients who responded to the survey, 57 patients (54.8\%) had intent to receive the COVID-19 vaccine. Vaccine efficacy, social responsibility, herd immunity, and desire to return to normal life were associated with selfreported willingness to receive a vaccine $(20.2 \%, 20.2 \%, 11.5 \%$, and $15.4 \%$, respectively). Unknown long-term safety, risk of adverse reaction to vaccine and concern that the vaccine is being developed too quickly were the most commonly reported reasons for non-uptake $(27.9 \%, 15.4 \%$, and $12.5 \%$, respectively).

Conclusion Half of the patients with inflammatory bowel disease would like to be vaccinated against SARS-CoV-2. This rate is similar to that reported in the French general population. Despite some concerns, patients with inflammatory bowel disease understood the necessity to be vaccinated against COVID-19.
\end{abstract}

Keywords Inflammatory bowel disease $\cdot$ Vaccination · COVID-19 - SARS-CoV-2

\begin{tabular}{|c|c|}
\hline \multicolumn{2}{|l|}{ Abbreviations } \\
\hline $\mathrm{CD}$ & Crohn's disease \\
\hline COPD & Chronic obstructive pulmonary disease \\
\hline COVID-19 & Coronavirus Disease 19 \\
\hline IBD & Inflammatory bowel disease \\
\hline IOIBD & $\begin{array}{l}\text { International organization for the study of } \\
\text { inflammatory bowel disease }\end{array}$ \\
\hline m-RNA & Messenger ribonucleic acid \\
\hline $\mathrm{R} \& \mathrm{D}$ & Research and development \\
\hline SARS-CoV-2 & $\begin{array}{l}\text { Severe Acute Respiratory Syndrome-Cor- } \\
\text { onavirus } 2\end{array}$ \\
\hline SD & Standard deviation \\
\hline
\end{tabular}

Laurent Peyrin-Biroulet

peyrinbiroulet@gmail.com

1 Department of Gastroenterology and Inserm NGERE U1256, Nancy University Hospital, University of Lorraine,

1 Allée du Morvan, 54511 Vandoeuvre-lès-Nancy, France $\begin{array}{ll}\text { TNF } & \text { Tumor necrosis factor } \\ \text { UC } & \text { Ulcerative colitis }\end{array}$

\section{Introduction}

The Coronavirus Disease 19 (COVID-19) is a systemic infection caused by the new Severe Acute Respiratory Syndrome-Coronavirus 2 (SARS-CoV-2), which has impacted on Health Systems, and has led to a dramatic shrinkage of the world economy [1]. The risk of COVID-19 in patients with inflammatory bowel disease (IBD) is similar to the general population [1-3]. In a large cohort, the cumulative incidence of SARS-CoV-2 infection in patients with IBD versus the general population was $0.406 \%$ and $0.402 \%$ cases, respectively [1]. Advances in age and treatment with corticosteroids impacted negatively on the outcome of COVID-19 [1]. 
Several SARS-CoV-2 vaccines are in advanced clinical development, and currently, there are few approved/awaiting approval [4]. At the beginning of December 2020, the first COVID-19 vaccine (Pfizer-BioNTech vaccine) was approved in the United Kingdom, then in the USA, and finally in the European Union [4]. Subsequently, a second m-RNA (messenger ribonucleic acid) vaccine (Moderna vaccine) and an inactive vaccine (AstraZeneca vaccine) have also been approved in a number of countries $[4,5]$. The British Society of Gastroenterology provided recommendations for the SARS-CoV-2 vaccination in IBD patients [6]. The International Organization for the Study of Inflammatory Bowel Disease (IOIBD) recommends that patients with IBD should be vaccinated against SARSCoV-2, and vaccination should not be deferred because a patient with IBD is receiving immune-modifying therapies [7].

Recently, one survey evaluated intention to receive a COVID-19 vaccine in two adult IBD populations (local and social media) [8]. This study identified a relatively high rate of COVID-19 vaccination intent (80.6\% for local and $61.1 \%$ for social media participants) [8]. However, limitations included selection and response bias inherent to online surveys [8].

In many countries, vaccine hesitancy and misinformation present substantial obstacles to achieving coverage and community immunity $[9,10]$. The accelerated pace of vaccine development has further heightened public anxieties and could compromise acceptance [11].

The aim of this study was to investigate the concerns, fears, and behaviors of patients with IBD about SARSCoV-2 vaccines.

\section{Materials and Methods}

\section{Study Participants}

The survey was implemented in routine practice and conducted from January 8th to February 22nd, 2021. One hundred and four consecutive ambulatory adult ( $>18$ years) patients with a proven diagnosis of IBD followed at the Nancy University hospital were included. Each patient received a questionnaire from their gastroenterologist in our unit. Answers were handwritten directly on the questionnaire by the patients, without receiving additional information. The data were entered into an anonymous electronic database.

\section{Data Collection}

We developed and administered a paper-based survey instrument. The questionnaire was developed in French, focusing on the most frequent questions asked by patients during daily practice. The questionnaire mainly consisted of multiplechoice questions and was designed to explore the following:

- Patients and IBD characteristics: age, gender, type and duration of IBD, age at diagnosis, phenotype, history of surgical treatment, disease activity, current medical IBD treatment, smoking status, comorbidities

- Personal history of infection with SARS-CoV-2, data about knowledge of COVID-19

- Vaccination perception, influenza, and pneumococcus vaccination status

- Data about knowledge of COVID-19 vaccination, intention regarding COVID-19 vaccination

\section{Analysis}

We analyzed the distribution of the responses against the different questions for the entire dataset. The data are expressed as numbers (\%) for qualitative data and as the means \pm standard deviations (SD) or medians \pm SD for qualitative data.

\section{Results}

\section{Study Population}

During the study period, a total of 104 patients with IBD were included. The characteristics of the study population are listed in Table 1 . The mean age at study inclusions was 42 years $(\mathrm{SD}=13.2)$, the mean age at IBD diagnosis was 26.5 years $(S D=12.2)$. Fifty patients $(48.1 \%)$ were male. Seventy-four percent of the patients (77/104) had Crohn's disease (CD), and $27 \%$ had ulcerative colitis (UC). The median duration of IBD was 11 years $(\mathrm{SD}=9.7)$. The majority of the patients were treated with biological agents, including anti-TNF (Tumor Necrosis Factor) in $40.4 \%$, vedolizumab in $12.5 \%$, and ustekinumab in $17.3 \%$. Eighteen patients $(17.3 \%)$ were not on any therapy for IBD. According to the patient global assessment, $62.5 \%$ of the patients considered themselves to be in clinical remission.

Twenty-two patients $(21.1 \%)$ were current smokers. Thirty patients (28.9\%) had comorbidities. Thirteen patients (12.5\%) had obesity. Seven patients (6.7\%) had hypertension, $2.9 \%$ of patients had diabetes mellitus, coronary artery disease was present in $3.8 \%$ of the patients, and one patient had stroke. Four patients (3.8\%) had chronic obstructive 
Table 1 Patient characteristics

\begin{tabular}{|c|c|}
\hline Characteristic & Total $(n=104)$ \\
\hline Male gender, $n(\%)$ & $50(48.1)$ \\
\hline Mean age, $y(\mathrm{SD})$ & $42(13.2)$ \\
\hline Current smoker, $n(\%)$ & $22(21.1)$ \\
\hline \multicolumn{2}{|l|}{ Age $(y)$ at diagnosis, $n(\%)$} \\
\hline$\leq 16$ & $7(6.7)$ \\
\hline $17-40$ & $80(77)$ \\
\hline$>40$ & $17(16.3)$ \\
\hline Mean time since diagnosis, $y$ (SD) & $11(9.7)$ \\
\hline \multicolumn{2}{|l|}{ Type of inflammatory bowel disease, $(\%)$} \\
\hline Crohn's disease & $77(74)$ \\
\hline Ulcerative colitis & $27(26)$ \\
\hline History of intestinal resection, $n(\%)$ & $36(34.6)$ \\
\hline Clinical remission & $65(62.5)$ \\
\hline \multicolumn{2}{|l|}{ Current treatment, $n(\%)$} \\
\hline None & $18(17.3)$ \\
\hline 5-ASA & $7(6.7)$ \\
\hline Budesonide & $1(1)$ \\
\hline Immunosuppressant & $8(7.7)$ \\
\hline Anti-TNF & $42(40.4)$ \\
\hline Vedolizumab & $13(12.5)$ \\
\hline Ustekinumab & $18(17.3)$ \\
\hline Investigational drugs & $2(1.9)$ \\
\hline \multicolumn{2}{|l|}{ Comorbidities } \\
\hline None & $74(71.1)$ \\
\hline Hypertension & $7(6.7)$ \\
\hline Diabetus mellitus & $3(2.9)$ \\
\hline Coronary heart disease & $4(3.8)$ \\
\hline Stroke & $1(1)$ \\
\hline Obesity & $13(12.5)$ \\
\hline COPD & $4(3.8)$ \\
\hline Asthma & $5(4.8)$ \\
\hline Cancer & $2(1.9)$ \\
\hline Renal disease & $1(1)$ \\
\hline Transplant & $1(1)$ \\
\hline
\end{tabular}

5-ASA 5-aminosalicylates, $C O P D$ chronic obstructive pulmonary disease, $S D$ standard deviation, $T N F$ tumor necrosis factor, $y$ years

pulmonary disease (COPD), $4.8 \%$ of the patients had asthma. Two patients had previous cancer, one patient had transplant, and one patient had renal disease.

\section{COVID-19 Perceptions}

We identified nine patients (8.6\%) who had either confirmed or highly suspected symptomatic COVID-19 infection. Eight patients had COVID-19 infection confirmed by a positive nasopharyngeal swab and one patient had highly suspected
Table 2 COVID-19 perceptions among patients with inflammatory bowel disease

\begin{tabular}{ll}
\hline Have you been infected with SARS-CoV-2? & 104 \\
Number of respondents & 8 \\
Confirmed & 1 \\
Highly suspected & 104 \\
Was someone in your family infected with SARS-CoV-2? & 41 \\
Number of respondents & \\
Yes & 104 \\
Does IBD predispose to COVID-19?* & 3.1 \\
Number of respondents & 3.2 \\
Average & \\
Standard deviation & 104 \\
Does IBD predispose to poor outcome of COVID-19?* & 3.8 \\
Number of respondents & 3.4 \\
Average & \\
Standard deviation & \\
Do the immunosuppressant/biologics predispose to poor out- & \\
come of COVID-19?* & \\
Number of respondents & 46 \\
Average & 4.5 \\
Standard deviation & 3.6 \\
\hline
\end{tabular}

COVID-19 (Coronavirus Disease 19), IBD (Inflammatory Bowel Disease),

SARS-CoV-2 (Severe Acute Respiratory Syndrome-Coronavirus 2)

*Respondents specified from 0 to 10 the risk

infection (Table 2). Forty-one patients had family members infected with SARS-CoV-2.

Patients were asked to quantify (from 0 to 10) the risk that patients with IBD could be infected with SARS-CoV-2. The average value was $3.1(\mathrm{SD}=3.2)$. They were asked to quantify the risk that patient with IBD and COVID-19 had poor outcome of COVID-19. The average value was 3.8 $(\mathrm{SD}=3.4)$. They were also asked to quantify the risk that patients with IBD and COVID-19 treated with immunosuppressant and/or biologics and/or small molecules had poor outcome of COVID-19. The average value was 4.5 $(\mathrm{SD}=3.6)$.

\section{Vaccination Perceptions}

Patients were asked to quantify (from 0 to 10 ) their view on recommended routine vaccinations. The average value was $7.6(\mathrm{SD}=3)$ (Table 3). Among the 104 patients, only onethird were up to date on influenza vaccine (35.6\%), 69.2\% for the pneumococcal vaccine. 
Table 3 Vaccination perceptions among patients with inflammatory bowel disease

\begin{tabular}{ll}
\hline In general, are you favorable of vaccination?* & \\
Number of respondents & 104 \\
Average & 7.6 \\
Standard deviation & 2.9 \\
Influenza vaccination status, $n(\%)$ & \\
Received influenza vaccination every year & $37(35.6)$ \\
Received influenza vaccination every few years & $14(13.5)$ \\
Never received influenza vaccination & $53(51)$ \\
Pneumococcus vaccination status, $n(\%)$ & \\
Vaccinated & $72(69.2)$ \\
Not vaccinated & $32(30.8)$ \\
\hline
\end{tabular}

*Respondents specified from 0 to 10 their opinion (0: negative opinion, 10: positive opinion)

\section{COVID-19 Vaccination Perceptions}

The majority of patients (86\%) agreed that people with CD or UC can have a COVID-19 vaccine (Fig. 1). Fifty-seven patients (54.8\%) had intent to receive the COVID-19 vaccine: $31 \%$ would certainly receive a COVID-19 vaccine, and $24 \%$ would probably receive a COVID- 19 vaccine.

The distribution of each perceptions item about COVID19 vaccine is presented in Table 4. Among patients who intended to get COVID-19 vaccine: $20.2 \%$ of patients believed that the vaccine protects against SARS-CoV-2 infection, $20.2 \%$ of patients wanted to protect people who might be vulnerable, $15.4 \%$ of patients desired to return to normal life, and $11.5 \%$ of patients believed in herd immunity. Eight patients $(7.7 \%)$ believed in favorable risk to benefit ratio, five patients $(4.8 \%)$ were at increased risk for complications of COVID-19 based on age or comorbidities, five patients $(4.8 \%)$ were confident in the scientists, and two patients (1.9\%) believed that COVID-19 vaccines had a promising safety profile.

Among patients who did not intent to get COVID-19 vaccine: $27.9 \%$ of patients believed that the long-term effects of vaccines were unknown, $15.4 \%$ of patients were afraid about the risk of adverse reaction to vaccine, and $12.5 \%$ of patients believed that the vaccine was being developed too quickly. Nine patients $(8.6 \%)$ were not confident in vaccine R\&D (Research and Development) process, six patients (5.8\%) had a personal history of allergic reactions, five patients (4.8\%) believed the vaccine will not work, four patients $(3.8 \%)$ were disappointed to have no choice between vaccines, and four patients $(3.8 \%)$ were not confident in pharmaceutical industry.

\section{Discussion}

Studies on the willingness of IBD populations to be vaccinated against COVID-19 are lacking. We investigated data from 104 patients with CD or UC followed at Nancy University hospital to identify their intention to receive a COVID-19 vaccine. Half of the patients had intent to receive the COVID-19 vaccine. This result is consistent with these in general population. In France, a study estimated of 54\% to $57 \%$ of the general population had COVID-19 vaccination intent [12]. For IBD patients, COVID-19 vaccine advantages are constituted not only by the individual and herd protection against SARS-CoV-2 but also by the desire to return to normal life. COVID-19 vaccine disadvantages are constituted by the risk of adverse reaction to vaccine, the unknown longterm safety, and the development of vaccines too quickly. A recent online survey of patients with IBD determined that $81 \%$ of local patients and $61 \%$ of social media patients in the United Kingdom had intent to receive the COVID-19 vaccine [8]. Patients who were hesitant to vaccination were largely concerned about long-term safety [8].

The results of this survey highlight that a gap between doctors and patients still exists. There is an urgent need to provide clear and specific recommendations in a period of a substantial confusion for people with chronic diseases. For adequate management of patients with IBD, it is essential that gastroenterologists are appropriately updated on efficacy and safety of the SARS-CoV-2 vaccine to provide clear information and guidance to patients with IBD, improving their attitude toward vaccination and reducing the skepticism and hesitation of some individuals [13]. In this context, patient associations can also be a key link between doctors and patients and should be increasingly involved in patient management. Close cooperation could allow greater patient compliance with the recommendations of health care providers.

Factors associated with the likelihood of accepting COVID-19 vaccination have been extensively studied in large population cohorts in order to drive public health information campaigns and to address vaccine hesitancy [14]. The role of the health care providers in recommending the vaccination results among the most significant factors in driving the compliance to vaccination [15]. This means that an essential task in reducing IBD patients' hesitancy to get vaccinated against COVID-19 will be played by gastroenterologists who will have to educate and inform their patients on the usefulness of vaccination, as already reported in the past for other vaccine recommendations [16].

A prospective cohort study evaluating the impact of a specialized infectious disease consultation on vaccination coverage rates in patients with gastrointestinal cancer or IBD showed this consultation could significantly improve 
Fig. 1 COVID-19 vaccination intent among patients with inflammatory bowel disease
Can people with Crohn's disease or ulcerative colitis have a COVID-19 vaccine?

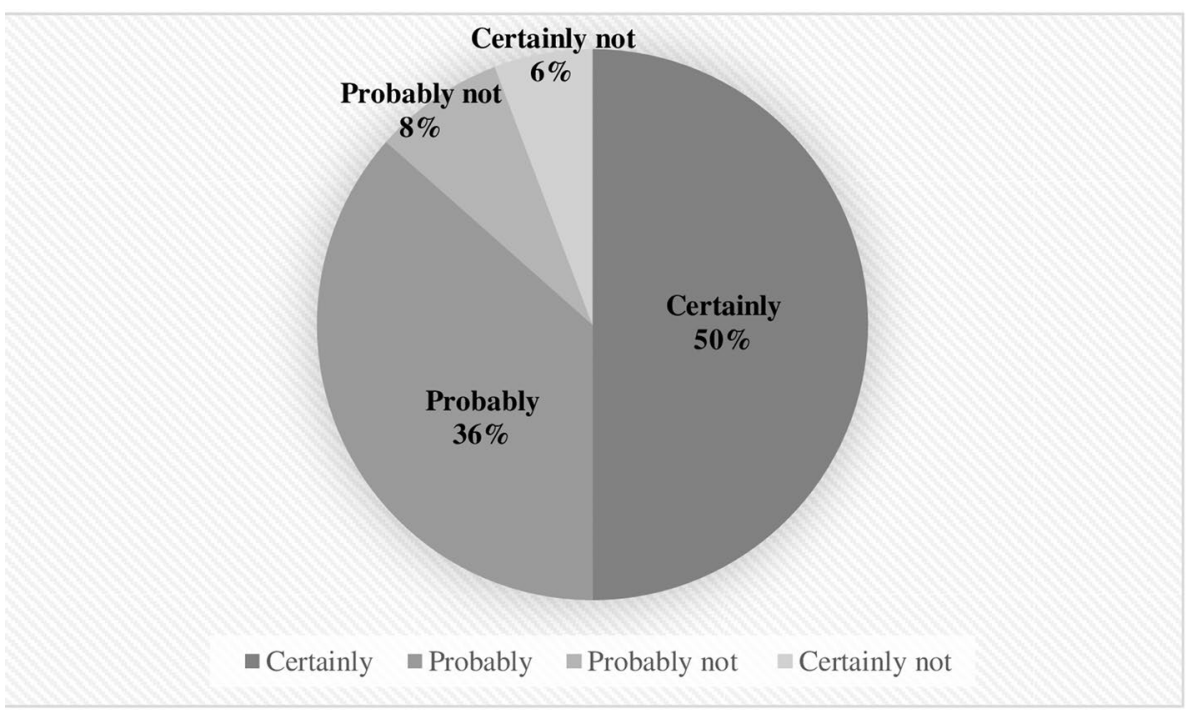

Do you intend to receive a COVID-19 vaccine?

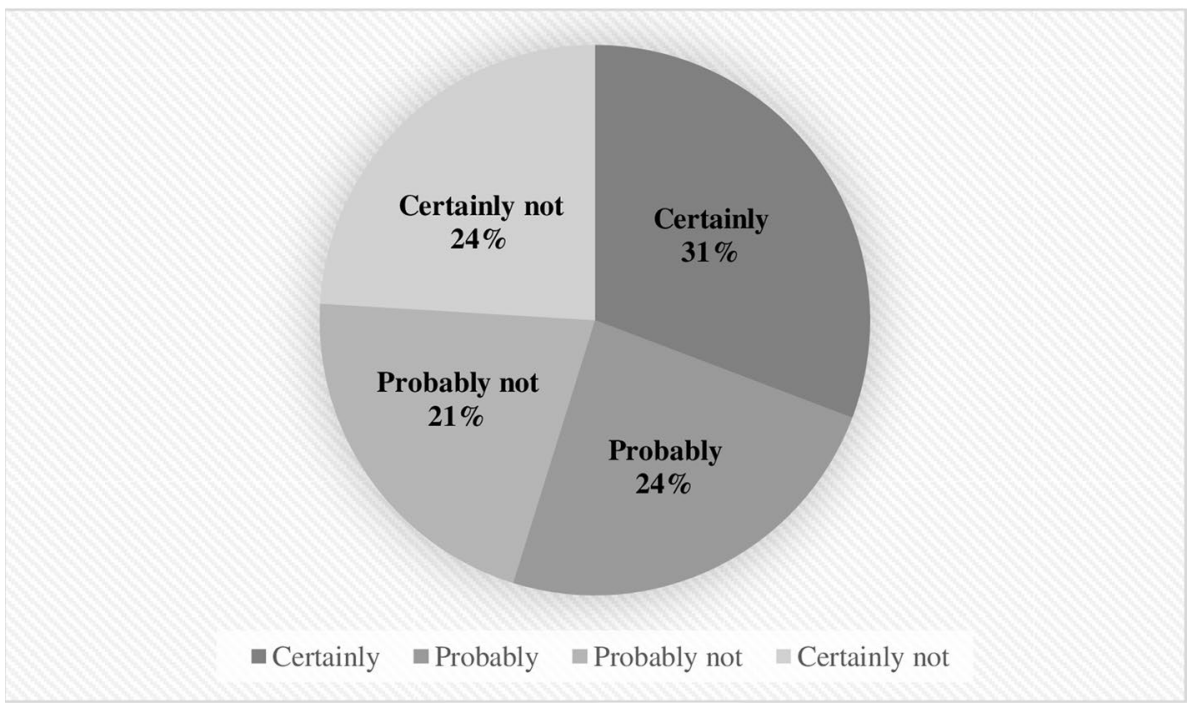

patients' knowledge about vaccination and vaccination coverage [17]. This approach could be applied to improve COVID-19 vaccination in IBD patients.

We also need to highlight some limitations of the present study. Most of the patients who answered the questionnaire were treated with biologics (72.1\%). We can suppose that this kind of patients has a more severe disease with more motivation to respect the recommendations. Future studies should also consider investigating if there are differences in COVID-19 vaccination rate in IBD patients between different countries.
In conclusion, our study suggests that COVID-19 vaccination intent among an IBD population is comparable with those found in the French general population. Patients with IBD should be vaccinated against SARS-CoV-2. The COVID-19 pandemic has presented several significant challenges including the willingness to be vaccinated against COVID-19. There is a need to highlight and emphasize the benefits of COVID-19 vaccination, which can reflect on strengthening trust in COVID-19 vaccines among the population. 
Table 4 Positive and negative attitudes toward COVID-19 vaccine

\begin{tabular}{ll}
\hline & Total $(n=104)$ \\
\hline Main reasons for intending to get COVID-19 vaccine, $n(\%)$ & $57(54.8)$ \\
Favorable risk to benefit ratio & $8(7.7)$ \\
Protection against SARS-CoV-2 infection & $21(20.2)$ \\
Promising safety profile & $2(1.9)$ \\
Social responsibility (to protect those who might be vulnerable) & $21(20.2)$ \\
Herd immunity & $12(11.5)$ \\
Individuals at increased risk for complications of COVID-19 based on age or comorbidi- & $5(4.8)$ \\
ties & \\
Desire to return to normal life & $16(15.4)$ \\
Confidence in the scientists & $5(4.8)$ \\
Main reasons for not intending to get COVID-19 vaccine, $n(\%)$ & $47(45.2)$ \\
Risk of adverse reaction to vaccine & $16(15.4)$ \\
Personal history of allergic reactions & $6(5.8)$ \\
The vaccine will not work & $5(4.8)$ \\
Not a member of any group that is at high risk for COVID-19 & $2(1.9)$ \\
Low risk to be infected with SARS-CoV-2 & $1(1)$ \\
Unknown long-term safety & $29(27.9)$ \\
Concern that the vaccine is being developed too quickly & $13(12.5)$ \\
Not confidence in vaccine R\&D process & $9(8.6)$ \\
No information about how long protection lasts after vaccination & $2(1.9)$ \\
No choice between vaccines & $4(3.8)$ \\
Don't trust the pharmaceutical industry & $4(3.8)$ \\
Doctor has not recommended a COVID-19 vaccine to me & $1(1)$ \\
\hline
\end{tabular}

COVID-19 Coronavirus Disease 19, $R \& D$ research and development, SARS-CoV-2 Severe Acute Respiratory Syndrome-Coronavirus 2

\begin{abstract}
Author's contributions $\mathrm{BC}$ contributed to data collection, wrote the article, and created tables and figures. EN contributed to data collection. LPB conceived the study, critically reviewed the content of the paper, and supervised the project. The manuscript was approved by all authors.
\end{abstract}

\section{Funding None.}

\section{Declarations}

Conflicts of interest B Caron and E Neuville declare no conflict of interest. L Peyrin-Biroulet has served as a speaker, consultant, and advisory board member for Merck, Abbvie, Janssen, Genentech, Mitsubishi, Ferring, Norgine, Tillots, Vifor, Hospira/Pfizer, Celltrion, Takeda, Biogaran, Boerhinger-Ingelheim, Lilly, HAC- Pharma, Index Pharmaceuticals, Amgen, Sandoz, For- ward Pharma GmbH, Celgene, Biogen, Lycera, Samsung Bioepis, Theravance.

\section{References}

1. Allocca M, Chaparro M, Gonzalez HA et al. Patients with inflammatory bowel disease are not at increased risk of COVID-19: a large multinational cohort study. J Clin Med 2020;9:3533.

2. Magro F, Rahier J-F, Abreu C et al. Inflammatory bowel disease management during the COVID-19 outbreak: the ten do's and don'ts from the ECCO-COVID taskforce. J Crohns Colitis 2020;14:S798-S806.

3. Allocca M, Fiorino $\mathrm{G}$, Zallot $\mathrm{C}$ et al. Incidence and patterns of COVID-19 among inflammatory bowel disease patients from the Nancy and Milan cohorts. Clin Gastroenterol Hepatol 2020;18:2134-2135.

4. Papa A, Gasbarrini A, Lopetuso LR. Winter is coming and COVID-19 vaccine is available! The role of gastroenterologist in increasing COVID-19 vaccine acceptability among IBD patients. Gastroenterology. 2021. https://doi.org/10.1053/j.gastro.2020.12. 066.

5. Voysey M, Clemens SAC, Madhi SA et al. Safety and efficacy of the ChAdOx1 nCoV-19 vaccine (AZD1222) against SARSCoV-2: an interim analysis of four randomised controlled trials in Brazil, South Africa, and the UK. The Lancet 2021;397:99-111.

6. The British Society of Gastroenterology. British Society of Gastroenterology Inflammatory Bowel Disease Section and IBD Clinical Research Group position statement on SARS-CoV2 Vaccination. January 4, 2021. https://www.bsg.org.uk/covid-19advice/british-society-of-gastroenterology-inflammatory-boweldisease-section-and-ibd-clinical-research-group-position-state ment-on-sars-cov2-vaccination/

7. Siegel CA, Melmed GY, McGovern DP, et al. SARS-CoV-2 vaccination for patients with inflammatory bowel diseases: recommendations from an international consensus meeting. Gut. https://doi.org/10.1136/gutjnl-2020-324000

8. Dalal RS, McClure E, Marcus J, et al. COVID-19 vaccination intent and perceptions among patients with inflammatory bowel 
diseases. Clin Gastroenterol Hepatol. https://doi.org/10.1016/j. cgh.2021.02.004

9. Larson HJ, Jarrett C, Eckersberger E et al. Understanding vaccine hesitancy around vaccines and vaccination from a global perspective: a systematic review of published literature, 20072012. Vaccine 2014;32:2150-2159.

10. Lane S, MacDonald NE, Marti M, Dumolard L. Vaccine hesitancy around the globe: analysis of three years of WHO/ UNICEF joint reporting form data-2015-2017. Vaccine 2018;36:3861-3867.

11. Fadda M, Albanese E, Suggs LS. When a COVID-19 vaccine is ready, will we all be ready for it? Int J Public Health 2020;65:711-712.

12. Santé Publique France. COVID-19 : point épidémiologique du 25 février 2021. Accessed March 3, 2021. https://www.santepubli quefrance.fr/maladies-et-traumatismes/maladies-et-infectionsrespiratoires/infection-a-coronavirus/documents/bulletin-natio nal/covid-19-point-epidemiologique-du-25-fevrier-2021.

13. D'Amico F, Rabaud C, Peyrin-Biroulet L, Danese S. SARS-CoV-2 vaccination in IBD: more pros than cons. Nat Rev Gastroenterol Hepatol. 2021. https://doi.org/10.1038/s41575-021-00420-w.
14. Lazarus JV, Ratzan SC, Palayew A et al. A global survey of potential acceptance of a COVID-19 vaccine. Nat Med 2021;27:225-228.

15. Reiter PL, Pennell ML, Katz ML. Acceptability of a COVID-19 vaccine among adults in the United States: How many people would get vaccinated? Vaccine 2020;38:6500-6507.

16. Coenen S, Weyts E, Jorissen C et al. Effects of education and information on vaccination behavior in patients with inflammatory bowel disease. Inflamm Bowel Dis 2017;23:318-324.

17. Sitte J, Frentiu E, Baumann C et al. Vaccination for influenza and pneumococcus in patients with gastrointestinal cancer or inflammatory bowel disease: a prospective cohort study of methods for improving coverage. Aliment Pharmacol Ther 2019;49:84-90.

Publisher's Note Springer Nature remains neutral with regard to jurisdictional claims in published maps and institutional affiliations. 